\title{
Performance Evaluation of Different Modulation Techniques for Different Mobility Pattern by using SVC Code over Wimax
}

\author{
Nancy \\ S.B.S.S.T.C Ferozepur
}

\author{
Chakshu Goel \\ S.B.S.S.T.C Ferozepur
}

\begin{abstract}
Wimax is Wireless Interoperability for Microwave Access for Wireless Communication IEEE 802.16e. In this paper the performance of different modulation techniques (QAM, adaptive and QPSK) by using different mobility patterns for VOD over Wimax with the help of VOD SVC coding. The work is done in terms of throughput, traffic sent and traffic received using OPNET modeler 14.5. Simulation shows that when nodes are moving through SVC coding in Wimax, the result is best and the overall performance of QPSK technique is superior to other techniques.
\end{abstract}

\section{Keywords}

Wimax, OPNET, Wireless Network, IEEE 802.16, VOD and SVC

\section{INTRODUCTION}

At present, remote innovation has turned into the most energizing at our encompassing surroundings; numerous sorts of techniques are utilized to make a communication through wireless system like Wimax, MANET, VANET, Wi-Fi, Bluetooth etc. According to expanding the notoriety of Broadband web remote systems administration, Wimax have utilized as a part of the field of wireless technology [2,3]. Wimax is Wireless Interoperability for Microwave Access; it is the most recent innovation for Wireless Communication which is in view of the IEEE 802.16-2004 and IEEE 802.16e2005 benchmarks and was outlined with much impact from Wi-Fi. IEEE 802.16 backings two sorts of transmission Duplexing: Time Division Duplexing (TDD) and Frequency Division Duplexing (FDD) and bolster both full and half duplex stations $[1,4,5]$.

Wimax is delineate the standards of orthogonal frequency division multiplexing (OFDM) which is a suitable modulation/access system for non-line-of-sight (LOS) conditions with high information rate [6,7].

\section{WIMAX STANDARDS}

The Wimax manages the IEEE 802.16 suite of principles. It characterizes inside its extension four PHY layers, any of which can be utilized with the media access control (MAC) layer to build up a broadband remote framework. The primary reason for IEEE 802.16 innovation was to give last-mile broadband remote access as a distinct option for link, advanced endorser line administration. The Wimax standard IEEE 802.16 is separated into taking after categories $[7,8,9$, 10]:

- 802.16: The initial 802.16 standard was discharged in December 2000. It gives a standard point-to multipoint telecast in 10 to $66 \mathrm{GHz}$ recurrence range for Line of Sight (LOS) environment [13, 15].
- 802.16a: The second form of Wimax standard 802.16 a was a correction of 802.16 norms and has the ability to show point-to-multipoint in the frequency range 2 to $11 \mathrm{GHz}$. It was secured in January 2003 and allocated both authorized and unlicensed recurrence groups. Unlicensed groups spread most extreme separation from 31 to 50 miles. It enhances the Quality of Service (QoS) characteristics with supporting conventions for occurrence Ethernet, ATM or IP [18,19].

- $\quad$ 802.16c: The third form of Wimax standard 802.16c was additionally a change of 802.16 norms which for the most part managed frequency ranging 10 to $66 \mathrm{GHz}$. This standard tended to different issues. For example, execution assessment, testing and itemized framework profiling. The framework profile is produced to indicate the obligatory gimmicks to guarantee interoperability and the optional features that separate items by valuing and usefulness $[17,19,20]$.

- 802.16d: In September 2003, an amendment venture known as $802.16 \mathrm{~d}$ started which meant to adjust to a specific perspective of European Telecommunications Standards Institute (ETSI). This undertaking was reasoned in 2004 with the arrival of 802.16d-2004 including all past Performance Evaluation of IEEE 802.16e (Mobile Wimax) in OFDM Physical Layer 25 adaptations changes. These standard backings obligatory and discretionary components alongside TDD and FDD technologies. Hypothetically, its successful information rate is $70 \mathrm{Mbps}$ yet in all actuality, the execution is close around $40 \mathrm{Mbps}$. This standard enhances the Quality of Service (QoS) by supporting substantial Service Data Units (SDU) and various surveying plans $[8,11]$.

- 802.16e: $802.16 \mathrm{e}$ was a correction of $802.16 \mathrm{~d}$ standard which completed in 2005 and known as $802.16 \mathrm{e}-2005$. Its principle point is portability including huge scope of scope. Infrequently it is called mobile WiMAX. This standard is a specialized overhaul of fixed WiMAX which has vigorous backing of portable broadband. Mobile Wimax was based on Orthogonal Frequency Division Multiple Access (OFDMA). It specified that, both measures (802.16d-2004 and 802.16e2005) backing the 256-FFT size. The OFDMA framework partitions signals into sub channels to broaden imperviousness to multipath obstruction. For example, if a $30 \mathrm{MHz}$ channel is isolated into 1000 sub-channels, every client would yield some 
sub-channels which are based on distance $[9,10$, $11]$.

\subsection{Fundamental Wimax Concepts}

WiMAX systems have five major design segments [5, 8, 11]:

- Base Station (BS): The BS is the hub that consistently joins wireless endorser gadgets to administrator systems. The BS keeps up communication with endorser gadgets and administers access to the administrator systems. A BS comprises of the foundation components important to empower wireless communications i.e., radio wires, handsets, and other electromagnetic wave transmitting gear. BSs are ordinarily settled hubs, yet they might likewise be utilized as a major aspect of portable answers for instance, a BS may be attached to a vehicle to give communications to close-by WiMAX gadgets.

- Subscriber Station (SS): The SS is a stationary WiMAX-proficient radio framework that communications with a base station.

- Mobile Station (MS): A MS is a SS that is planned to be utilized while as a part of movement at up to vehicular paces. Contrasted and altered (stationary) SSs, MSs ordinarily are battery worked and in this way utilize improved force administration. Illustration MSs incorporate WiMAX radios installed in laptops and cellular telephones.

- Relay Station (RS): RSs are SSs designed to forward movement to different RSs or SSs in a multi-hop Security Zone. The RS may be in an altered area (e.g., connected to a building) or portable (e.g., set in a vehicle).

- Operator Network: The administrator system envelops infrastructure network functions that give radio access and IP network administrations to WiMAX subscribers. These capacities are characterized in WiMAX Forum specialized particulars as the entrance administration system (radio access) and the integration administration system (IP connectivity).

\section{MODULATION TECHNIQUES}

\section{- Quadrature Phase Shift Keying (QPSK):}

It is otherwise called four-level PSK where every component speaks to more than one bit. Every symbol contains two bits and it utilizes the stage movement of $\pi / 2$, methods $90^{\circ}$ as opposed to moving the phase $180^{\circ}$. In this mechanism, the group of stars comprises of four focuses however the choice is constantly made in two bits. This mechanism can guarantee the proficient utilization of bandwidth and higher unearthly proficiency $[15,17,20]$.

- Quadrature Amplitude Modulation (QAM):

This is the most mainstream balance procedure utilized as a part of different wireless standards. It consolidated with ASK and PSK which has two separate signs sent simultaneously on the same frequency yet one ought to be shifted by $90^{\circ}$ as for the other signal. At the receiver end, the signals are demodulated and the outcomes are joined to get the transmitted binary data $[12,13]$.

\section{- 64 QAM:}

This is same as 16 QAM aside from it has 64-states where every symbol represents six bits $\left(2^{6}=64\right)$. It is a complex modulation technique however with greater efficiency. The aggregate data transfer capacity increments as per the expanding number of states for every symbol. Mobile WiMAX utilizes this higher modulation technique when the connection condition is high $[11,12,13]$.

\section{VIDEO ON DEMAND}

Video on demand $(\mathrm{VoD})$ is an interactive TV technology that allows subscribers to view programming in real time. Video on demand is one of the dynamic features offered by Internet Protocol TV. The video data is transmitted by means of RealTime Streaming Protocol. VoD is also very commonly used for videoconferencing. VoD can work well over a wide geographic region or on a satellite-based network as long as the demand for programming is modest. VoD provides a wide selection of video programming including sports, entertainment, educational programs and feature films. In general, TV is based on broadcast technology, while VoD is provided as a unicast transmission.

\section{SCALABLE VIDEO CODING}

SVC standardizes the encoding of a high-quality video bit stream that also contains one or more subset bit streams. A subset video bit stream is derived by dropping packets from the larger video to reduce the bandwidth required for the subset bit stream.SVC has achieved significant improvements in coding efficiency with an increased degree of supported scalability relative to the scalable profiles of prior video coding standards. Codec's are used to compress video to reduce the bandwidth required to transport streams or to reduce the storage space required to archive them.

\section{EXPERIMENTAL SETUP}

In this experiment the Effect of Mobility patterns of Mobile WiMAX Subscriber on Video on Demand (VoD) over WiMAX is analyzed by using OPNET Simulator for different modulation techniques. OPNET Simulator 14.5 was used to analyze the performance of Wimax. We used OPNET modeler, as OPNET modeler provides a comprehensive development environment supporting the modeling of communication network and distributed systems.

OPNET modeler provides better environment for simulation, data collection and data analysis. In this experiment different scenarios are taken.

In these scenarios Subscriber is moving with different mobility patterns. In first scenario the mobility pattern of nodes is random, in second scenario the mobility pattern of nodes is along the trajectory and in third scenario some nodes are fixed and some nodes are moving randomly. These scenarios are repeated for different modulation techniques (QAM, QPSK and adaptive). To compare this SVC code is used.

In each scenario 7 Hexagonal cells are taken. Each cell has a radius of $2 \mathrm{Km}$. In each cell there is one Base station and 15 mobile nodes. These nodes are circularly placed. The BS connected to the IP backbone via a DS3 WAN link. The node 0 is connected to backbone through ppp-sonet-oct1 link. The node is also connected to video server through 100 base $\mathrm{T}$ Ethernet link.

\section{RESULTS}

Here results of different modulation techniques for different mobility patterns are given:

1. Throughput: Throughput refers to how much data can be transferred from one location to another in a given amount of time. 
sample_sum (in WiMAX.Throughput (bits/sec))

nancy project1-fixed 64-DES-1 nancy project1-qam 64 any-DES-1 nancy project1-qam 64 tra-DES-1

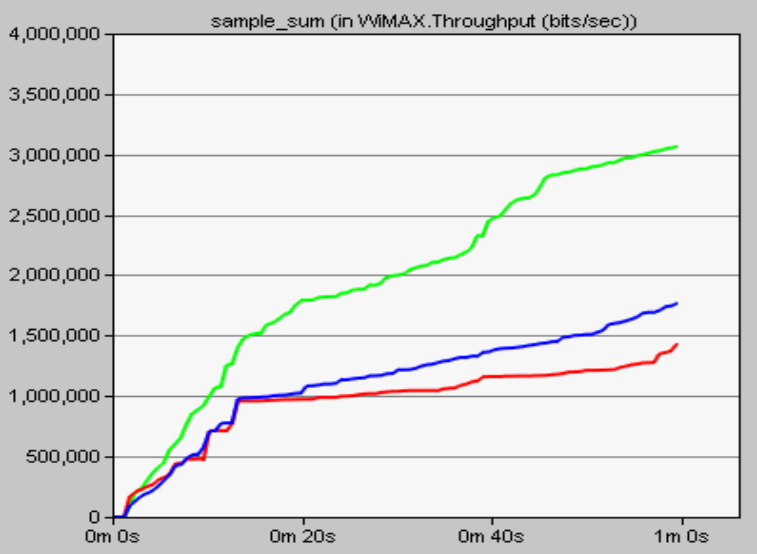

Fig 1: Qam 64 for different Mobility patterns

Fig 1 shows that when the nodes are moving according to our trajectory throughput is more which is 3100000 . When the some nodes move and some are fixed than the throughput is 1600000 and when it is moving randomly than throughput is 1400000. These result shows that the performance of QAM under according to our route is better than other

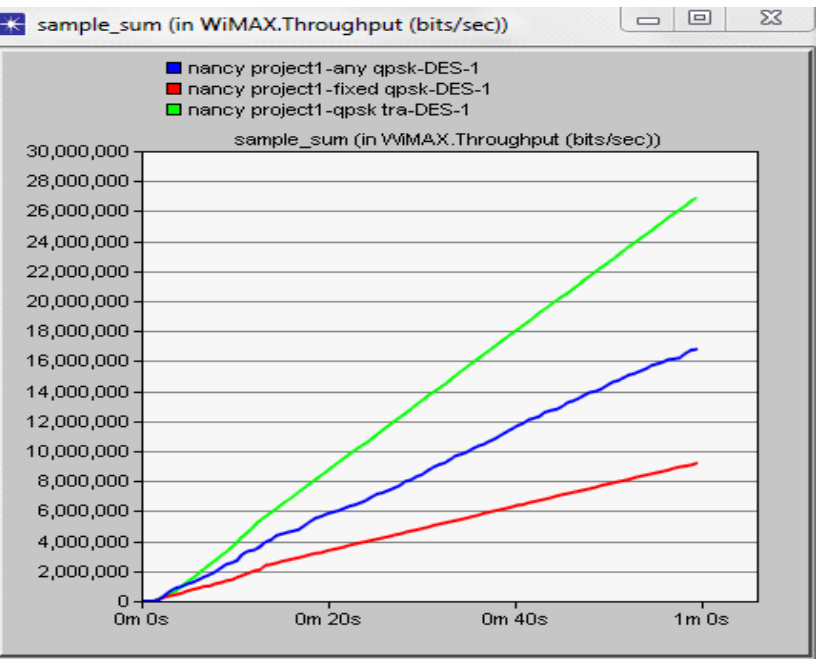

\section{Fig 2: QPSK for different Mobility patterns}

Fig 2 shows that when the nodes are moving according to our trajectory throughput is more which is 27000000 . When the some nodes move and some are fixed than the throughput is 17000000 and when it is moving randomly than throughput is 1000000 . For throughput the result is better when the nodes are moving under given way than other mobility pattern

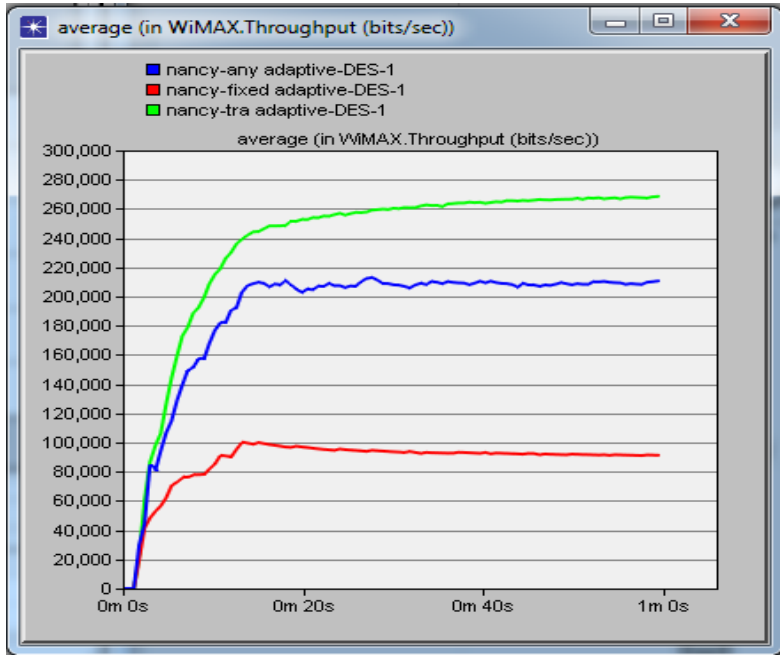

Fig 3: Adaptive for different Mobility patterns

Fig 3 shows that when the nodes are moving according to our trajectory throughput is more which is 270000 . When the some nodes move and some are fixed than the throughput is 100000 and when it is moving randomly than throughput is 210000. For throughput the result is better when the nodes are moving under given way than other mobility pattern.

\section{Traffic Received}

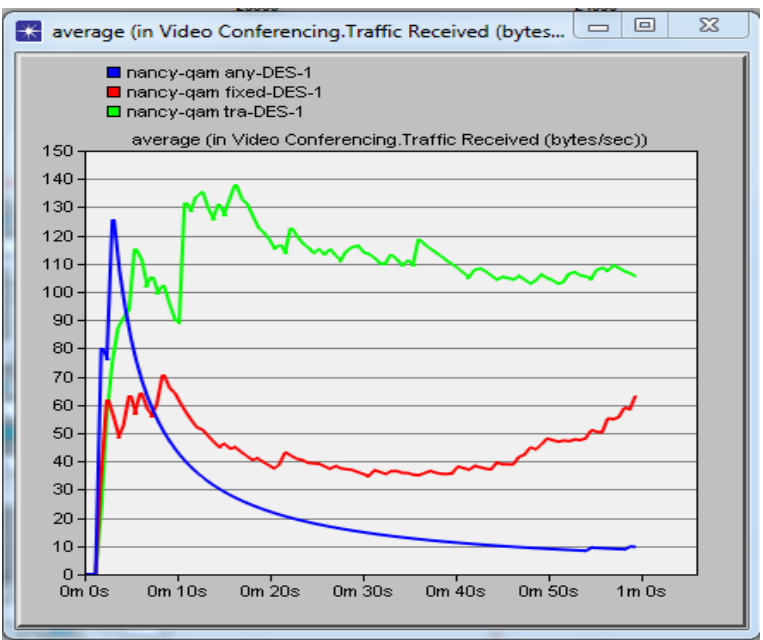

Fig 4: Traffic received for Mobility patterns using 64QAM3/4

Fig 4 shows that when the nodes are moving on the way which is given by us the traffic received is 110 . When they are moving and some are fixed then the traffic received are 70 and when they are moving anywhere then the traffic received is 10. The result shows that when then nodes are moving under given way then the result is better than other mobility pattern. 


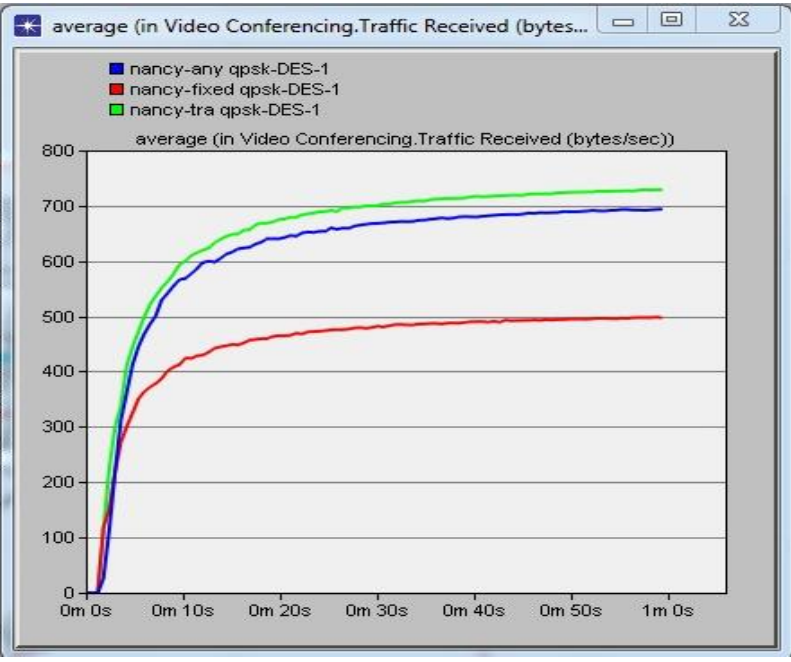

Fig 5: Traffic received for Mobility patterns using QPSK

Fig 5 shows that when the nodes are moving on the way which is given by us the traffic received is 750 . When they are moving and some are fixed then the traffic received are 500 and when they are moving anywhere then the traffic received is 700. The result shows that when then nodes are moving under given way then the result is better than other mobility pattern.

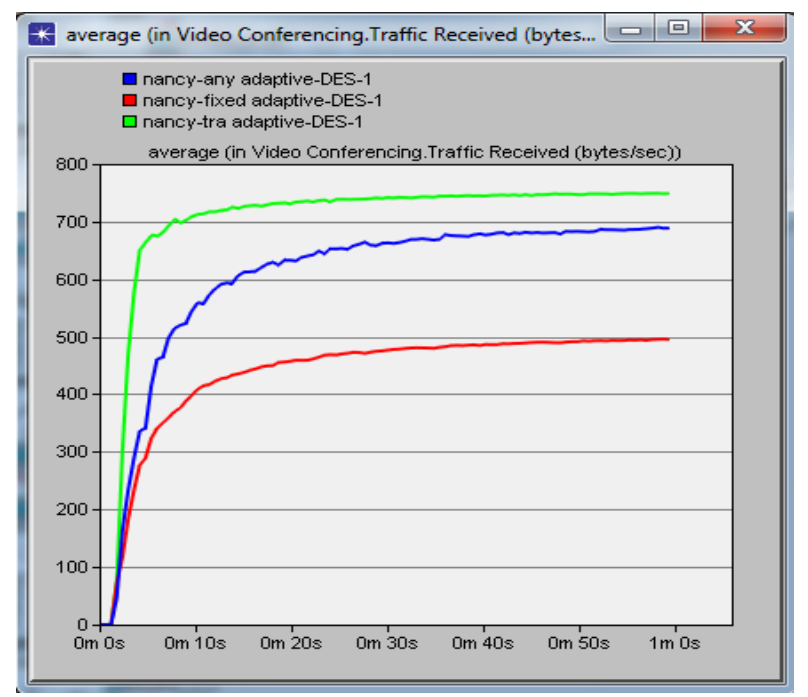

Fig 6: Traffic received for Mobility patterns using Adaptive

Fig 6 shows that when the nodes are moving on the way which is given by us the traffic received is 760 . When they are moving and some are fixed then the traffic received are 500 and when they are moving anywhere then the traffic received is 690. The result shows that when then nodes are moving under given way then the result is better than other mobility pattern.

\section{Traffic Sent}

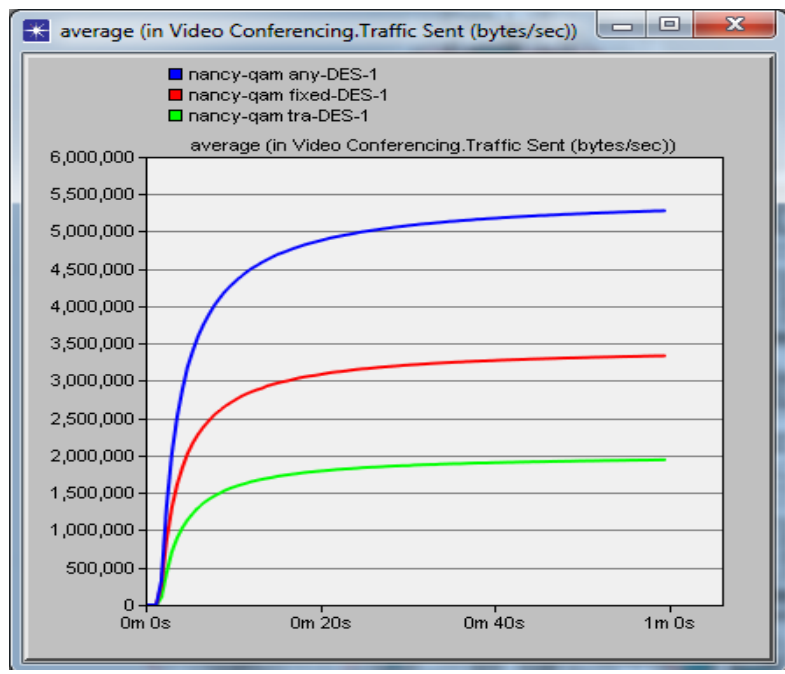

Fig 7: Traffic sent for Mobility patterns using 64QAM3/4

Fig 7 shows that when the nodes are moving on the way which is given by us the traffic sent is 5300000 . When they are moving and some are fixed then the traffic sent is 3400000 and when they are moving anywhere then the traffic sent is 2000000. The result shows that when then nodes are moving any way then the result is better than other mobility pattern

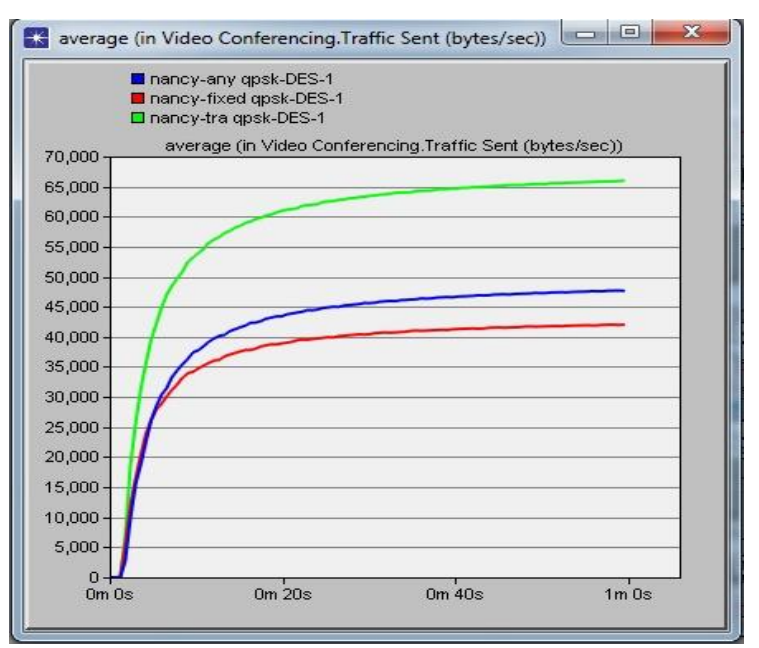

Fig 8: Traffic sent for Mobility patterns using QPSK

Fig 8 shows that when the nodes are moving on the way which is given by us the traffic sent is 65000 . When they are moving and some are fixed then the traffic sent is 42000 and when they are moving anywhere then the traffic sent is 48000 . The result shows that when then nodes are moving under given way then the result is better than other mobility pattern 


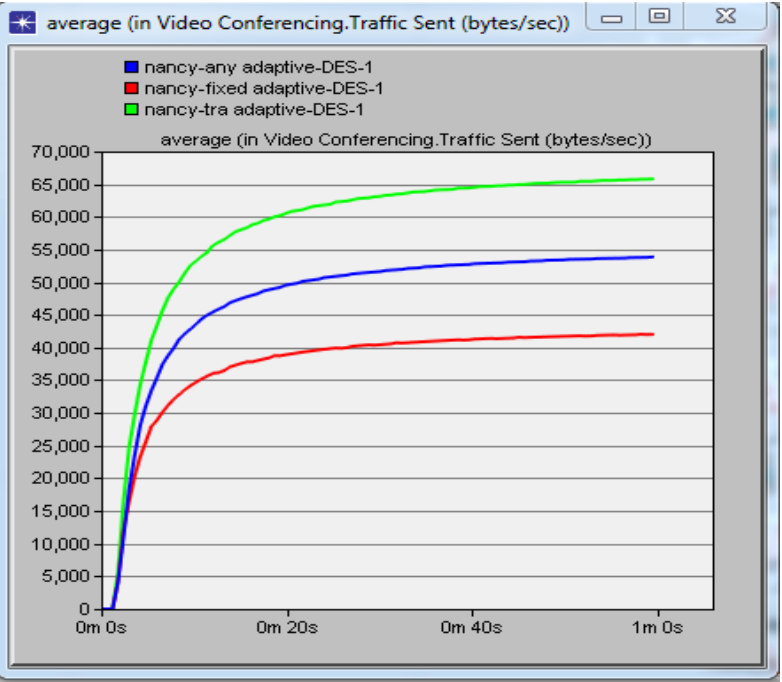

Fig 9: Traffic sent for Mobility patterns using Adaptive

Fig 9 shows that when the nodes are moving on the way which is given by us the traffic sent is 66000 . When they are moving and some are fixed then the traffic sent is 42000 and

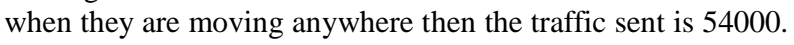
the result shows that when then nodes are moving under given way then the result is better than other mobility pattern.

\section{CONCLUSION}

In this research analysis of the performance of IPTV (VoD) over Wimax by varying Mobility pattern of Mobile Wimax Subscriber in terms of Traffic Received, Throughput and Traffic Sent is carried out for different modulation techniques. Its aim is to address the performance metrics of QoS for video streaming when deploying over WiMAX access technology. The OPNET Modeler is used to design and characterize the performance parameters of SVC video streaming with different modulation techniques to WiMAX video subscribers using QoS performance metrics for different mobility pattern of nodes. In this experiment the placement of nodes are circular within hexagonal cell of radius $2 \mathrm{~km}$. For video streaming SVC codes are used. Simulation is carried out for one minute. The results showed that when nodes are moving in a given way the result is best, the result also shows that the performance of QPSK technique is better than other techniques. In Future we can do this work by good and bad factors and by applying different mobility pattern on nodes.

\section{REFERENCES}

[1] Ghosh A. Wolter, D.R., "Broadband wireless access with Wimax/802.16: current performance benchmarks and future potential", Communications Magazine, IEEE (Volume:43, Issue: 2 ),2005.

[2] Hung-Yu Wei, Ganguly S., "Interference-aware IEEE 802.16 WiMax mesh networks" Vehicular Technology Conference. VTC-Spring. IEEE 61st (Volume:5),2005.

[3] Chakchai So-In, Jain, R., "Scheduling in IEEE 802.16e mobile WiMAX networks: key issues and a survey," IEEE Journal on (Volume:27, Issue: 2 ),2009.

[4] Deb, S. Bell Labs, "Real-Time Video Multicast in WiMAX Networks", INFOCOM The 27th Conference on Computer Communications. IEEE ,2008.

[5] Schwarz, H.,Heinrich Hertz, "Overview of the Scalable Video Coding Extension of the H.264/AVC Standard"
,Circuits and Systems for Video Technology, IEEE Transactions on (Volume:17, Issue: 9, 2007.

[6] Pentikousis, K. Pinola, J., “An experimental investigation of VoIP and video streaming over fixed WiMAX" , Modeling and Optimization in Mobile, Ad Hoc, and Wireless Networks and Workshops, 2008.

[7] Will Hrudey, "Streaming Video Content Over IEEE 802.16 / WiMAX Broadband Access", Spring 2008.

[8] Sharangi, S. Krishnamurti, R., "Energy-Efficient Multicasting of Scalable Video Streams Over WiMAX Networks" Multimedia, IEEE Transactions on (Volume:13, Issue: 1 ) 2010.

[9] Yong Liu, Yang Guo, Chao Liang, "A survey on peerto-peer video streaming systems" peer-to-Peer Networking and Applications, Volume 1, Issue 1, pp 1828, 2008.

[10] Y. Wang, “ Survey of Objective Video Quality Measurements, Tech report," Worcester Polytechnic Institute, June 2006.

[11] C. Cicconetti, C. Eklund, L. Lenzini, E. Mingozzi, “ Quality of service support in IEEE 802.16 Networks", IEEE Network 20 (2006).

[12] J. Casasempere, P. Sanchez, "Performance evaluation of H.264/MPEG-4 Scalable Video Coding over IEEE 802.16e networks"IEEE International Symposium on, 13-15 May 2009.

[13] Mengke Hu, Hongguang Zhang, “Performance evaluation of video streaming over mobile WiMAX networks" , GLOBECOM Workshops (GC Wkshps), 2010 IEEE, vol., no., pp.898-902, 6-10 Dec. 2010.

[14] S. Winkler, P. Mohandas, "The evolution of video quality measurement: from PSNR to hybrid metrics", IEEE Transactions on Broadcasting 54 (2008).

[15] T. Wiegand, G.J. Sullivan, G. Bjontegaard, "Overview of the h.264/avc video coding standard", IEEE Transactions on Circuits and Systems for Video Technology (2003).

[16] M. Chatterjee, S. Sengupta, and S. Ganguly, "FeedbackBased real-time streaming over WiMax," IEEE Wireless Communications Magazine, vol. 14, no. 1, pp. 64-71, Feb. 2007.

[17] H. Juan, H. Huang, C. Huang, and T. Chiang, "Scalable video streaming over mobile WiMAX," Proc. ISCAS 2007, New Orleans, Louisiana, May 2007, pp. $3463-$ 3466.

[18] F. Yousaf, K. Daniel, and C. Wietfeld, "Performance evaluation of IEEE 802.16 WiMAX link with respect to higher layer protocols," Proc. IEEE ISWCS 2007, Trondheim, Norway, Oct. 2007, pp. 180-184.

[19] F. Retnasothie, M. Ozdemir, T. Yucek, H. Celebi, J. Zhang, and R. Muththaiah, "Wireless IPTV over WiMAX: challenges and applications," Proc. IEEE WAMICON 2006, Clearwater, FL, Dec. 2006, pp. 1-5.

[20] H. Schulzrinne, A. Rao, and R. Lanphier, "Real Time Streaming Protocol (RTSP)", IETF RFC 2326, April 1998.

[21] OPNET official website, http://www.opnet.com. 